\title{
Prognostic factors and efficacy of GDP-R therapy in refractory/relapsed diffuse large B-cell lymphomas not eligible for high-dose therapy
}

\author{
Francesco Ghio ${ }^{1}$, Giulia Cervetti ${ }^{1}$, Nadia Cecconi ${ }^{1}$, Matteo Pelosini ${ }^{1}$, Sara Galimberti ${ }^{1}$, Riccardo Morganti ${ }^{2}$, \\ Paola Ferrari' ${ }^{1}$, Andrea Nicolini' ${ }^{1}$, Mario Petrini ${ }^{1}$ \\ ${ }^{I}$ Department of Clinical and Experimental Medicine, University of Pisa, 67-56127 Pisa, Italy. \\ ${ }^{2}$ Department of Oncology, University Hospital of Pisa, 67-56127 Pisa, Italy.
}

Correspondence to: Dr. Francesco Ghio, Department of Clinical and Experimental Medicine, University of Pisa, 67-56127 Pisa, Italy.

E-mail: francescoghio83@gmail.com

\section{A B S T R A C T}

Aim: The main aim of the present study was to evaluate the overall survival (OS) and time to treatment failure (TTF) in a cohort of relapsed/refractory diffuse large B-cell lymphomas (DLBCLs) not eligible for high-dose therapy (HDT) treated with gemcitabine in association with dexamethasone, cisplatin and rituximab (GDP-R) protocol. The secondary aim was to identify the prognostic factors impacting OS and TTF. Methods: The authors retrospectively analyzed 45 patients with refractory/relapsed DLBCLs treated with GDP-R. Results: Overall response rate (ORR) was 48.8\%; complete response 15/45 (33.3\%), partial response 7/45 (15.5\%). Response was influenced by the number of previous therapies administered and International Prognostic Index (IPI) value. Although no significant impact occurred with regard to OS, patients pre-treated with 2 or $<2$ chemotherapeutic regimens had better ORR $(P=0.014)$ and a longer TTF $(P=0.029$ in multivariate Cox model). IPI value also influenced TTF. Patients with $<$ 2 IPI value had significantly more prolonged TTF than the other ones $(P=0.048$ in multivariate Cox model). Treatment was welltolerated, with the majority of patients treated on out-patient modality. GDP-R regimen represents a valid treatment for aggressive relapsed/refractory B-cell lymphoma not eligible for HDT thanks to its efficacy and good toxic profile. Conclusion: The number of previous chemotherapeutic regimens and IPI value select those who benefit more from this treatment.

Key words: Cisplatin; dexhametazone; GDP; gemcitabine; relapsed/refractory diffuse large B-cell lymphomas

\section{INTRODUCTION}

Diffuse large B-cell lymphomas (DLBCLs) are quite often curable with intensive combination chemotherapy. Despite the improvement of outcome with chemoimmunotherapy, $30-40 \%$ of patients relapse after the first-line treatment, and the rate of the second complete remission is lower than $30 \% \cdot{ }^{[1-3]}$ Management of these cases is not well-established. High-dose therapy (HDT) with hematological stem-cell support is the standard treatment for chemosensitive patients. Induction salvage therapies are usually based on platinum and etoposide: R-DHAP (rituximab, dexamethasone, cytosine arabinoside, and cisplatin) and R-ESHAP (rituximab, etoposide, methylprednisone, Ara-C, and cisplatin) are generally used, ${ }^{[4]}$ but they are often characterized by poor responsiveness and significant toxicity. Gemcitabine, an antimetabolite drug, has shown

\begin{tabular}{|l|l|}
\hline \multicolumn{2}{|c|}{ Access this article online } \\
\hline Quick Response Code: & Website: \\
\hline & www.jcmtjournal.com \\
\cline { 2 - 3 } & \\
\hline
\end{tabular}

significant activity in heavily pre-treated patients with NHL even after autologous stem cell transplantation (ASCT). Its favorable toxicity profile allows its use in combination regimens with other cytotoxic drugs and anti-CD20targeted therapy with an overall response rate (ORR) of $50-60 \%$ in different phase II studies. ${ }^{[5-9]}$ In the present retrospective study, we described the experience of our institution about the use of gemcitabine in association with cisplatin, dexamethasone, and rituximab (GDP-R), in relapsed/refractory DLBCLs not eligible for (HDT) with hematological stem cell support. The principal aims of this study were to evaluate the overall survival (OS) and treatment failure (TTF) and the prognostic factors impacting OS and TTF.

This is an open access article distributed under the terms of the Creative Commons Attribution-NonCommercial-ShareAlike 3.0 License, which allows others to remix, tweak, and build upon the work non-commercially, as long as the author is credited and the new creations are licensed under the identical terms.

For reprints contact: service@oaepublish.com

How to cite this article: Ghio F, Cervetti G, Cecconi N, Pelosini M, Galimberti S, Morganti R, Ferrari P, Nicolini A, Petrini M. Prognostic factors and efficacy of GDP-R therapy in refractory/relapsed diffuse large B-cell lymphomas not eligible for high-dose therapy. J Cancer Metastasis Treat 2016;2:59-63.

Received: 09-07-2015; Accepted: 24-11-2015. 


\section{METHODS}

\section{Patients}

From February 2006 to July 2014, 45 relapsed/ refractory DLBCLs patients treated with GDP-R at our Institution entered into the study. Eligibility criteria were men or women aged $>18$ years; documentation of unresponsiveness disease according to the Cheson criteria, ${ }^{[10]}$ after one or more chemotherapeutic regimens; absence of renal, hepatic, and respiratory failure; no evidence of active infections; HIV-negativity; at least one site of measurable disease; and written informed consent. In particular, of a total of 45 studied patients, $37(82 \%)$ relapsed after achieving an initial complete response (CR), while the remaining 8 patients $(18 \%)$ were primary nonresponders (primary refractory disease). Patient evaluation included a full history and clinical examination, complete serum biochemistry with dosage of lactate dehydrogenase (LDH) and $\beta 2$-microglubulin, peripheral blood and bone marrow immunophenotyping, bone marrow biopsy, bone marrow molecular analysis, chest and abdomen and pelvic computed tomographic scan, serology for HIV, hepatitis-B virus and hepatitis- $\mathrm{C}$ virus. The age range of the cohort was 23-84 years. The number of previous therapies (NPTs) was also evaluated in this series. The first-line chemotherapy was R-CHOP $(n=35)$, R-DHAP $(n=2)$, and hyper-CVAD $(n=8)$. Most cases $(27 / 45)$ received less than two previous chemotherapies; 20/45 cases had bone marrow involvement documented by biopsy (stage IV).

\section{Treatment}

GDP-R regimen consisted of gemcitabine $\left(1,000 \mathrm{mg} / \mathrm{m}^{2}\right)$ intravenous (IV) on the days 1 and 8 ; cisplatin $\left(75 \mathrm{mg} / \mathrm{m}^{2}\right)$ IV on the day 1 ; rituximab $375 \mathrm{mg} / \mathrm{m}^{2}$ IV on the day 2 ; oral dexamethasone $40 \mathrm{mg}$ on the days $1-4$; this regimen was given every 3 weeks for a total of four courses. The standard anti-emetic regimen including ondansetron and dexamethasone was provided prior to chemotherapy. Chemotherapy was delayed on day 8 until recovery for a maximum of 3 weeks if the neutrophil count was $<0.5$ $\times 10^{9} / \mathrm{L}$ and $/$ or the platelet count was $<50 \times 10^{9} / \mathrm{L}$ or if the patient showed grade $3 / 4$ non-hematological toxicity (except for nausea, vomiting, and alopecia). The dose of cisplatin was reduced by $50 \%$ in the event of grade 2 neurological toxicity or grade 1 renal toxicity. In the event of febrile neutropenia, grade 4 thrombocytopenia or more than grade 3 non-hematological toxicity (except alopecia), treatment with $75 \%$ of the dose was given. Patient's disease was evaluated for response 1 month after the end of treatment, and then every 3 months during the first 2 years and every 6 months for further 3 years. International Workshop NHL response criteria were used to assess the response to treatment. ${ }^{[10]}$ The toxicity was estimated and graded according to the National Cancer Institute Common Toxicity Criteria version 3.0 grading system. Side-effects were described in the overall population and in each of 2 subsets that were divided according to the International Prognostic Index (IPI) value and numbers of chemotherapeutic regimens as prognostic factors referred to OS and TTF.

\section{Statistical analysis}

Before performing survival analysis, an exploration phase was carried out. Categorical data were described by frequency and percentage, whereas continuous data by mean, median, and range.

\section{Complete and partial response to chemotherapy}

$\mathrm{CR}$ and partial response (CR and PR, respectively, according to the Cheson criteria) in patients with more than 2 or 2 or $<2$ chemotherapeutic regimens were assessed by using the Fisher exact test.

\section{Survival analysis}

The survival was expressed as mean, median, and range. The endpoints studied included TTF (defined as the time from the beginning of treatment to further disease progression, relapse, or death) and OS (defined as the time from diagnosis to the last follow-up). Six variables (risk factors) were assessed in TTF and OS univariate and multivariate survival analysis: sex (male, female); age $(\leq 65,>65)$; LDH $(\leq 300,>300)$; stage (I-II, III-IV); IPI: $(\leq 2,>2)$; and NPT $(\leq 2,>2)$.

The results of the Cox regression were expressed using both the hazard ratios with its related confidence interval and related $P$ value.

Survival curves were calculated using the Kaplan-Meier method and the log-rank test was used to evaluate the differences between curves. Univariate survival analysis was performed including each risk factor in a Cox regression model. All variables significantly influencing survival in the univariate analysis were analyzed together in a Cox regression model as multivariate analysis, with the aim of studying the independent contribution of each risk factor in explaining survivorship. Furthermore, the proportional hazard is always verified by using of $\log (-$ $\log$ ) curves. The results of the Cox regression were expressed by hazard ratios with its related confidence interval and related $P$ value calculated by Wald test. Regression coefficients (B) were also calculated. Statistics was applied to the overall population $(n=45)$ and to each of the two subsets that were obtained after all patients were divided according to whether the IPI value was $\leq 2$ or $>2$ and the number of chemotherapeutic regimens was $\leq 2$ or $>2$ ( $27 v s .18$ pts, respectively). The cut-off value for the number of previous chemotherapies and IPI was determined by a preliminary investigation considering the available data from the study.

Differences were considered significant at $P<0.05$.

Analyses were performed using the SPSS 21 technology. 


\section{RESULTS}

The principal clinical characteristics of patients are shown in Table 1. All studied patients had received 2 previous chemotherapeutic programs as median (range: 1-5). All cases were evaluable for response. ORR was $48.8 \%$ : CR 15/45 (33.3\%); PR 7/45 (15.5\%). At the time of this analysis, after a median follow-up of 22 months (range: 5-148), 4/22 responsive patients relapsed with a median duration of response of 10.5 months (range: 4-15). With a median followup of 57 months, the 2-year TTF and OS rates were $43 \%$ and $70 \%$, respectively. No significant difference occurred with regard the OS in the 2 subsets divided according to the IPI value and numbers of chemotherapeutic regimens $(P$ $=0.823$ and $P=0.389$, respectively) [Table 2]. Response was influenced by the NPTs. Of 45 patients, 27 were pretreated with 2 or less than 2 chemotherapeutic regimens and 12 achieved CR, 5 PR, and 10 a stable/progressive disease (SD/PD), with an ORR of 17/27 (63\%). The remaining 18 patients were pre-treated with more than 2 chemotherapeutic regimens. Three cases of them obtained a CR, one a PR, and the 14 remaining an SD/PD with an ORR of 4/18 (22\%). Thus, patients pre-treated with 2 or $<2$ chemotherapeutic regimens had better ORR ( $P=0.014$, Fisher exact test). TTF

Table 1: Principal clinical characteristics of patients

\begin{tabular}{|c|c|c|}
\hline & Number & $\%$ \\
\hline \multicolumn{3}{|l|}{ Sex } \\
\hline Female & 20 & 44 \\
\hline Male & 25 & 56 \\
\hline \multicolumn{3}{|l|}{ Age } \\
\hline$\leq 65$ years & 36 & 80 \\
\hline$>65$ years & 9 & 20 \\
\hline \multicolumn{3}{|l|}{ LDH } \\
\hline$\leq 300 \mathrm{UI} / \mathrm{L}$ & 18 & 40 \\
\hline$>300 \mathrm{UI} / \mathrm{L}$ & 27 & 60 \\
\hline \multicolumn{3}{|l|}{ Stage } \\
\hline I & 2 & 4 \\
\hline II & 12 & 26 \\
\hline III & 11 & 25 \\
\hline IV & 20 & 45 \\
\hline \multicolumn{3}{|l|}{ IPI } \\
\hline 0 & 2 & 4 \\
\hline 1 & 9 & 20 \\
\hline 2 & 16 & 36 \\
\hline 3 & 15 & 34 \\
\hline 4 & 1 & 2 \\
\hline 5 & 0 & 0 \\
\hline Not available & 2 & 4 \\
\hline \multicolumn{3}{|l|}{ NPT } \\
\hline 1 & 9 & 20 \\
\hline 2 & 18 & 40 \\
\hline 3 & 13 & 29 \\
\hline 4 & 4 & 9 \\
\hline 5 & 1 & 2 \\
\hline
\end{tabular}

NPT: number of previous treatments; LDH: lactate dehydrogenase; IPI: international prognostic index median time was 22.2 months for patients pre-treated with 2 or $<2$ chemotherapeutic regimens and 2.7 months for the other ones [Figure 1] $[P=0.029$ in multivariate analysis; Table 3]. Even IPI value was able to influence TTF: patients with IPI $\leq 2$ had significantly more prolonged TTF than the other ones [ $P=0.048$ in multivariate analysis; Table 3].

\section{Toxicity}

No serious adverse event was observed. The treatment was generally well-tolerated, with the majority of patients treated on out-patient modality. Neutropenia grades 2, 3, and 4 were, respectively, reported in $8.9 \%, 4.4 \%$, and $2.2 \%$ of cases; whereas thrombocytopenia grades 2 and 3 were reported in $4.4 \%$ and $8.8 \%$ of patients, respectively. No febrile neutropenia was observed. Grade 2 neurotoxicity occurred in $2.2 \%$, but no grade $3 / 4$ neurotoxicity was reported. In 6 patients, creatinine levels (which not overcame $176 \mu \mathrm{mol} / \mathrm{L}$ ) increased during treatment. Hospitalization was necessary in 1 case. As to toxicity not significant difference occurred in each subset of patients and it was not affected by the number of previous treatments. In fact, among 27 patients pre-treated with 2 or $<2$ chemotherapeutic regimens, we recorded 8 cases of hematological toxicity (29\%) and in the remaining 18 patients treated with more than 2 chemotherapeutic regimens, we recorded 5 hematological toxicity $(28 \%)(P=\mathrm{ns})$.

\section{DISCUSSION}

About $40-60 \%$ of elderly patients with DLBCL will be refractory or will experience relapse during their clinical course. ${ }^{[1]}$ These and other patients are not eligible for ASCT due to old age, or important comorbidities and management of this population is not yet standardized. Many current regimens, such as DHAP, ICE, ESHAP, show an ORR between $39 \%$ and $69 \%$, but remarkable side-effects are frequent. ${ }^{[3,4]}$ Therefore, these regimens are not feasible for this subset of refractory/relapsed DLBCLs. Gemcitabine is a drug classified as a

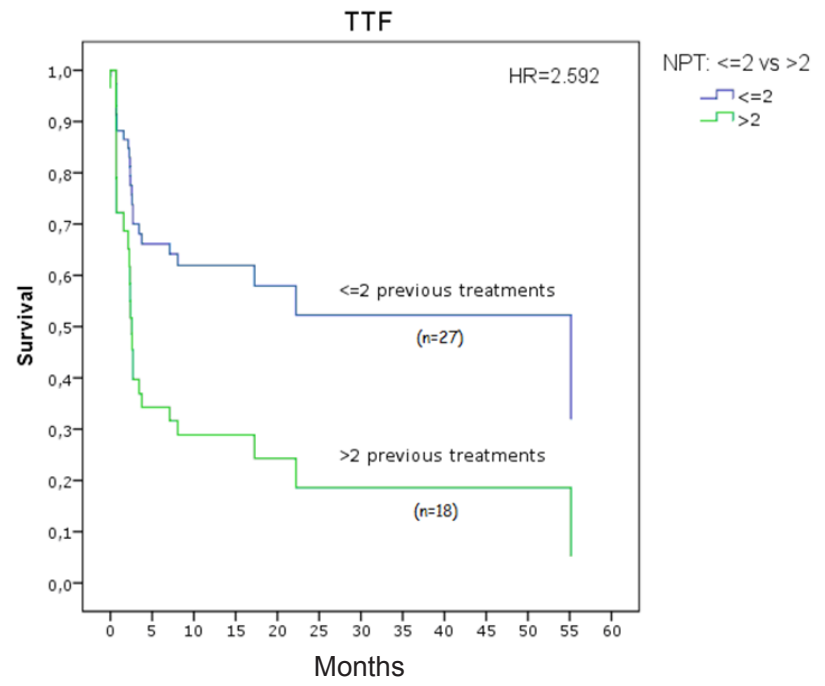

Figure 1: Time to treatment failure curves according to the number of previous chemotherapeutic regiments 
Table 2: OS risk factors

\begin{tabular}{lccc}
\hline & \multicolumn{3}{c}{ Univariate analysis } \\
\cline { 2 - 4 } & $\boldsymbol{P}$ & HR & IC 95\% \\
\hline Sex & 0.555 & 1.31 & $0.53-3.24$ \\
Age & 0.289 & 1.74 & $0.62-4.85$ \\
LDH & 0.271 & 1.80 & $0.63-5.12$ \\
Stage & 0.863 & 0.91 & $0.32-2.62$ \\
IPI & 0.823 & 1.15 & $0.34-3.92$ \\
NPT & 0.389 & 1.40 & $0.60-3.76$ \\
\hline
\end{tabular}

NPT: number of previous treatments; LDH: lactate dehydrogenase; IPI: international prognostic index; OS: overall survival

Table 3: TTF risk factors

\begin{tabular}{|c|c|c|c|c|c|c|c|}
\hline & \multicolumn{3}{|c|}{ Univariate analysis } & \multicolumn{4}{|c|}{ Multivariate analysis } \\
\hline & $P$ & HR & IC95\% & B & $P$ & HR & IC95\% \\
\hline Sex & 0.551 & 1.28 & $0.57-2.87$ & & & & \\
\hline Age & 0.536 & 1.37 & $0.51-3.66$ & & & & \\
\hline LDH & 0.290 & 1.59 & $0.67-3.76$ & & & & \\
\hline Stage & 0.243 & 1.80 & $0.67-4.85$ & & & & \\
\hline IPI & 0.041 & 2.82 & $1.01-7.87$ & 1.29 & 0.048 & 3.65 & $124-10.7$ \\
\hline NPT & 0.019 & 2.59 & $1.17-5.74$ & 1.15 & 0.029 & 3.17 & $1.12-8.95$ \\
\hline
\end{tabular}

NPT: number of previous treatments; LDH: lactate dehydrogenase; IPI: international prognostic index; TTF: time to treatment failure

nucleoside analog. It is a competitive substrate with deoxycytidine for incorporation into DNA, and in this way, it inhibits DNA replication and repair. It is a derivative of cytidine and even if it is similar to cytosine arabinoside, it can be absorbed by cells faster, more effectively phosphorylated, and it remains in cells for a longer of time. Gemcitabine inhibits the DNA synthesis by preventing the activity of ribonucleotide reductase, and this conduces to a reduction of the concentration of intracellular nucleotide pool. In this way, gemcitabine has more antitumor activities and a lighter bone marrow inhibition than higher dosage of cytosine arabinoside. ${ }^{[5,6]}$ As far as we know, one previous study ${ }^{[12]}$ only has been conducted using the same GDP-R regimen of chemoimmunotherapy in patients with refractory/relapsed DLBCLs as in our report. In this study, in 50 successive patients, the 2-year OS and progression-free survival were $70 \%$ and $48 \%$, respectively. Hence, both these end-points was the same or similar to those we have reported in our study. ORR was $72 \%$ and grade III-IV neutropenia and thrombocytopenia occurred in 34\% and $40 \%$ of patients. However, the schedule adopted by the investigators in this study was different than in ours. In fact, cisplatin was given at $25 \mathrm{mg} / \mathrm{m}^{2} \mathrm{IV}$ on the days $1-3$ instead of $75 \mathrm{mg} / \mathrm{m}^{2}$ on the day 1 and rituximab was delivered on the day 1 instead of on the day 2. These slight differences could have affected both ORR and toxicity that were higher than in our study. Moreover, another previous study evaluated the efficacy of GDP regimen given with the same schedule as in our study but not including rituximab. ${ }^{[13]}$ In this study, the ORR was $58.3 \%$ for assessable patients, and the 1 -year OS rate was $41.7 \%$.
This last value is much lower than that we have reported at 2 -year in our study $(70 \%)$ and suggests that the addition of rituximab to GDP regimen significantly increases its efficacy. However, an occurrence rate for grade III/IV leukopenia of $37.5 \%$ and $25 \%$ for thrombocytopenia was found. These rates are higher than those we have observed in our study and the reason is not clear. In our study, 45 patients with aggressive refractory/relapsed DLBCLs not eligible for ASCT were treated with GDP-R achieving an ORR of $48.8 \%$ with a median duration of response of 13.59 months (range: 2.13-58.6 months). Moreover, GDP-R resulted safe: no febrile neutropenia was recorded; grade-4 neutropenia was registered in one patient, and two patients developed grade- 2 neurotoxicity. These data confirm GDP-R therapy is a reasonable option for refractory/relapsed DLBCLs in patients who are not eligible for ASCT. In particular, patients pre-treated with 2 or $<2$ lines of therapy had a better ORR than that of ones $(63 \% v s .22 \%)$ receiving more than 2 lines before GDP-R, with a median TTF of 22.2 months vs. 2.7 months $(P=0.029$ in multivariate Cox model). Even IPI influenced TTF with a median of 17.3 months for patients with IPI value less or equal to 2 and 3.4 months for patients with IPI $>2(P=0.048$ in multivariate Cox model). These data suggest that exposition to numerous different chemotherapeutic regimens selects chemoresistant neoplastic cells that are difficult to be eradicated. Moreover, they suggest that within the entire population of patients with refractory/relapsed DLBCLs not eligible for ASCT the number of previous chemotherapeutic regimens and IPI value select those who benefit more from GDP-R treatment. It is likely that the disease was intrinsically more aggressive in patients with higher IPI index and in those who required multiple chemo-treatments. In fact, tumor phenotype and its biological aggressiveness are different in any cancer. In the multivariate analyses, among the evaluated prognostic factors, the number of previous chemo-treatments and IPI index were significant variables for TTF. This finding suggests that the number of previous chemo-treatments and IPI are independent prognostic factors. Moreover, tumor phenotype can change during the progression of the disease due to genetic instability of cancer cells. This could account for the lack of a significant correlation between the number of previous chemo-treatments or IPI and OS. In fact, prognostic factors other than the number of previous chemo-treatments and IPI and inherent to tumor phenotype can prevail with the progression of the disease.

In conclusion, the shown results, even if based on a retrospective monocentric study and a small sample size, evidence that for patients with relapsed/refractory DLBCL, who cannot benefit from HDT and GDP-R is a reliable and well-tolerated therapeutic choice. 


\section{Financial support and sponsorship}

Nil.

\section{Conflicts of interest}

There are no conflicts of interest.

\section{REFERENCES}

1. Armitage JO. A clinical evaluation of the International Lymphoma Study Group classification of non-Hodgkin's lymphoma. Blood 1997;89:3909-18.

2. Groves FD, Linet MS, Travis LB, Devesa SS. Cancer surveillance series: non-Hodgkin's lymphoma incidence by histologic subtype in the United States from 1978 through 1995. J Natl Cancer Inst 2000;92:1240-51.

3. Gisselbrecht C, Glass B, Mounier N, Singh Gill D, Linch DC, Trneny M, Bosly A, Ketterer N, Shpilberg O, Hagberg H, Ma $\mathrm{D}$, Brière $\mathrm{J}$, Moskowitz $\mathrm{CH}$, Schmitz N. Salvage regimens with autologous transplantation for relapsed large B-cell lymphoma in the rituximab era. J Clin Oncol 2010;28:4184-90.

4. Sweetenham JW, Johnson PW. ESHAP chemotherapy for relapsed/ refractory non-Hodgkin's lymphoma. J Clin Oncol 1994;12:2766.

5. Bernell $\mathrm{P}, \mathrm{Ohm} \mathrm{L}$. Promising activity of gemcitabine in refractory highgrade non-Hodgkin's lymphoma. Br J Haematol 1998;101:203-4.

6. Fossa A, Santoro A, Hiddemann W, Truemper L, Niederle N, Buksmaui S, Bonadonna G, Seeber S, Nowrousian MR. Gemcitabine as a single agent in the treatment of relapsed or refractory aggressive non-Hodgkin's lymphoma. J Clin Oncol 1999;17:3786-92.
7. Santoro A, Bredenfeld H, Devizzi L, Tesch H, Bonfante V, Viviani S, Fiedler F, Parra HS, Benoehr C, Pacini M, Bonadonna G, Diehl V. Gemcitabine in the treatment of refractory Hodgkin's disease: results of a multicenter phase II study. J Clin Oncol 2000;18:2615-9.

8. Savage DG, Rule SA, Tighe M, Garrett TJ, Oster MW, Lee RT, Ruiz J, Heitjan D, Keohan ML, Flamm M, Johnson SA. Gemcitabine for relapsed or resistant lymphoma. Ann Oncol 2000;11:595-7.

9. Crump M, Baetz T, Couban S, Belch A, Marcellus D, HowsonJan K, Imrie K, Myers R, Adams G, Ding K, Paul N, Shepherd L, Iglesias J, Meyer R. Gemcitabine, dexamethasone, and cisplatin in patients with recurrent or refractory aggressive histology B-cell non-Hodgkin lymphoma: a phase II study by the National Cancer Institute of Canada Clinical Trials Group (NCIC-CTG). Cancer 2004;101:1835-42.

10. Cheson BD, Horning SJ, Coiffler B, Shipp MA, Fisher RI, Connors JM, Lister TA, Vose J, Grillo-López A, Hagenbeek A, Cabanillas F, Klippensten D, Hiddemann W, Castellino R, Harris NL, Armitage JO, Carter W, Hoppe R, Canellos GP. Report of an international workshop to standardize response criteria for non-Hodgkin's lymphoma. J Clin Oncol 1999; 17:1244.

11. Martelli M, Ferreri AJ, Agostinelli C, Di Rocco A, Pfreundschuh M, Pileri SA. Diffuse large B-cell lymphoma. Crit Rev Oncol Hematol 2013;87:146-71.

12. Hou Y, Wang HQ, Ba Y. Rituximab, gemcitabine, cisplatin, and dexamethasone in patients with refractory or relapsed aggressive B-cell lymphoma. Med Oncol 2012;29:2409-16.

13. Fan Y, Huang ZY, Luo LH, Yu HF. Efficacy of GDP regimen (gemcitabine, dexamethasone, and cisplatin) on relapsed or refractory aggressive non-Hodgkin's lymphoma: a report of 24 cases. Ai Zheng 2008;27:1222-5. 